\title{
A Practical Method to Hourly Forecast the Solar Irradiance
}

\author{
Tao Hai ${ }^{\mathrm{a}}{ }^{*}$, Kewei Wen $^{\mathrm{b}}$, Jian Zhong $^{\mathrm{c}}$, Xiang Hu${ }^{\mathrm{c}}$, and Zhao Zhang ${ }^{\mathrm{d}}$ \\ Institute of Electrical Engineering,Guangxi University,Nanning 530004,Guangxi,China \\ ahaitao5913@163.com, bwkw712123@163.com, chuxiang1122@sina.cn, d850395420@qq.com
}

Key Words: solar irradiance, neural network, hourly prediction, UV index, theoretical extraterrestrial irradiance

\begin{abstract}
Based on the fact that the weather bureau currently does not provide solar irradiance forecast data, which is a key parameter to predict energy where photovoltaic (PV) is generated, a practical and indirect solar irradiance prediction model that is based on the RBF neural network and input by several of hourly sequences is proposed. In this paper, the model is built by using UV index sequence, theoretical extraterrestrial irradiance sequence, sequence of air temperature, sequence of weather types and historical solar irradiance sequence. The network is trained by GP-RBF algorithm to forecast the solar irradiance in a period of time to the future by dividing conditions into four types. The experiment results show that, when compared with the other forecasts and the real curves, the new model, which is based on UV index and theoretical extraterrestrial irradiance hourly sequences, is practical and highly accurate.
\end{abstract}

\section{Introduction}

PV power generation plays a prominent role in the reduction of environmental pollution, improvement of energy structure, and the relief of the energy crisis and other aspects, which has become an important direction in renewable energy utilization. However, the solar irradiance is the major factor that affect PV power generation, resulting in instability and randomness that makes the photovoltaic power intermittently and volatile. Large-scale PV grid connected to the management and safety of power system brings problems [1], and it's extremely important for the prediction of PV power generation and its plan, operation and control to forecast solar irradiance. Now all the countries in the world are to explore ways to predict the intensity of solar radiation accurately. At present, short-term researches on the method for prediction of solar irradiance are mainly about regression analysis, time series and neural network which has better prediction ability at home and abroad [2]. Because now it is difficult to get the direct and short-term prediction of solar irradiance, in this paper, a practical, indirect prediction model based on RBF neural network and hourly solar irradiance of the input sequence is proposed.

\section{The general idea of solar irradiance forecast}

Because ultraviolet rays are a part of the solar radiation, usually when the solar radiation is intense, so are the ultraviolet rays, and the different weather types can reflect different solar radiation intensities. The UV index and types of weather forecast data reflect different seasons, different weather types of solar irradiance. Thus, we use the UV index and weather types forecasted to indirectly predict solar irradiance [3].

Extraterrestrial solar irradiance reflects the rotation and revolution information of the earth, can also be inputting constraint, and by hourly weather we can get the weather type sequence and 
temperature series and UV index sequence forecasted in next day, so it doesn't matter to build prediction model by sorting the season and weather patterns. Hourly weather data can be acquired in the www.accuweather.com. Due to the fact that night solar irradiance is 0 , in this method, the predicted sequence from 6 a.m. to 7 p.m. is considered only in a local day in order to reduce the calculation amount [4].

Extraterrestrial solar irradiance refers to the theoretical strength solar radiation not by any occlusion and reflection has arrived at the earth's surface strength theory, the theory of extraterrestrial solar irradiance in the accurate calculation day of prediction at $6 \mathrm{a} . \mathrm{m}$. to 7 p.m. is to ensure the solar irradiance forecast accuracy [5].

$$
E_{0}=E_{s c} \times(\bar{R} / R)^{2} \times \sinh
$$

In Eq. 1, $E_{s c}$ refers to the solar constant, $E_{s c}=1366.1 \mathrm{~W} / m^{2}, R$ refers to the sun earth distance, $\bar{R}$ refers to the correction factor of the sun earth distance, $\mathrm{h}$ refers to the Angle of the sun.

\section{Establish the forecasting model}

The RBF neural network is a kind of three layer forward network, which is composed of the signal source nodes, whose topological structure is shown in Fig. 1. The transformation function of the hidden layer unit is a non-negative nonlinear function of the local distribution, and the radial symmetry of the central point is attenuated. The unit number of the hidden layer is determined by the need to describe the problem. The output of the network is the linear weighted sum of the output of the hidden units. The input space of the RBF neural network is nonlinear, and the transformation from the hidden layer to the output layer is linear [6].

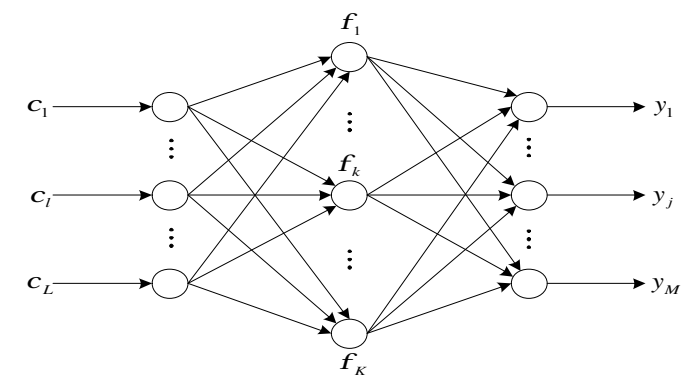

Fig.1. the RBF neural network

The basic idea of RBF neural network is the radial basis function which is used as the basis of the hidden layer unit to compose the hidden layer space. The radial basis function of RBF neural network is usually used as the Gauss function shown in Eq. 2. The output of the RBF network is a linear combination of the hidden layer nodes which is shown in Eq. 3.

$$
\phi_{\mathrm{j}}=\exp \left(-\frac{\left\|x-c_{j}\right\|^{2}}{\sigma_{j}^{2}}\right), j=1,2 ; \cdot \cdot \mathrm{N}_{\mathrm{h}}
$$

In Eq. $2,{ }_{\mathrm{j}}$ refers to the output of the first $\mathrm{j}$ hidden layer nodes, $\mathrm{x}=\left[\mathrm{x}_{1}, \mathrm{x}_{2}, \cdots, \mathrm{x}_{\mathrm{n}}\right]^{\mathrm{T}}$ refers to the input sample, ${ }^{c_{\mathrm{j}}}$ refers to the Center of Gauss's functions, ${ }^{\sigma_{\mathrm{j}}}$ refers to the Width of Gauss's function, $N_{\mathrm{h}}$ refers to the hidden layer node number. 


$$
\mathrm{y}_{\mathrm{i}}=\sum_{j=1}^{N_{h}} \omega_{i j} \phi_{j}=W_{\mathrm{i}}^{T} \Phi, i=1,2, \cdots, m
$$

In Eq. $3, W_{\mathrm{i}}=\left[\omega_{i 1}, \omega_{i 2}, \cdots, \omega_{i N_{h}}\right]^{T}, \Phi=\left[\phi_{1}, \phi_{2}, \cdots, \phi_{N_{h}}\right]^{T}$.

At this stage, there are lots of solar irradiance prediction models of neural network shown in Fig. 2 (a). When selecting input variables, more considerations are paid to historical solar irradiance sequence, temperature, season and weather type in the forecasting day without taking the degree of solar radiation and most directly related to the parameters into account [7]. Its main features are shown as below:

(1) It is necessary to set up the forecast model by sorting the season and weather patterns, which is simple and the forecast accuracy is not high.

(2) The solar irradiance for each time, only becomes overall larger or smaller, cannot predict solar irradiance when weather suddenly changes.

(3) Just to reflect changes in solar irradiance by season and weather patterns

(4) There is no restriction on the input.

(5) The input data of the meteorological is a single value, rather than an hour series input

For the faults of non-hourly input sequence prediction model, a practical hourly input sequence prediction model is proposed, as shown in Fig. 2 (b). We select input variables, add temperature series, weather type sequence, UV index sequence and extraterrestrial solar irradiance in the forecasting day. Its main features are shown as below:

(1)The forecasting sub models are established not by the season and weather patterns, but by using hourly weather forecast data.

(2) Reflect the changes of solar irradiance by weather types and UV index.

(3) Able to predict the weather the change of solar irradiance

(4) Join the extraterrestrial solar irradiance theory as a restrictive condition prediction

(5) The input vector and the output vector are both the sequence of the hour parameter

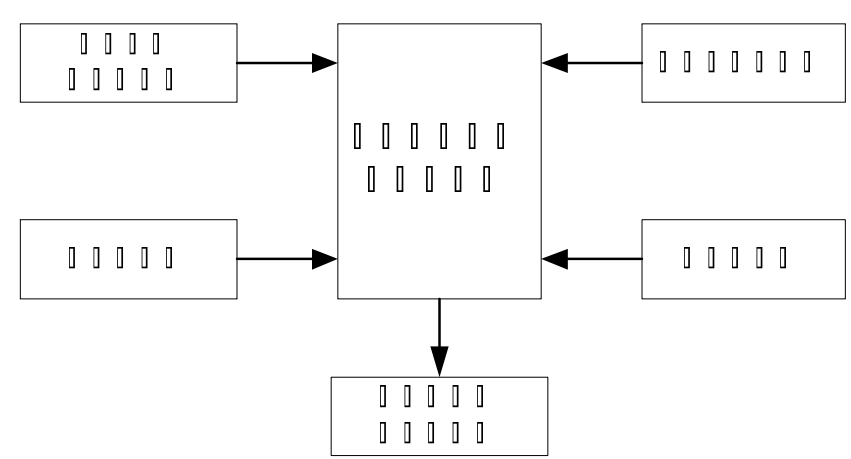

( a ) Non time by time series input

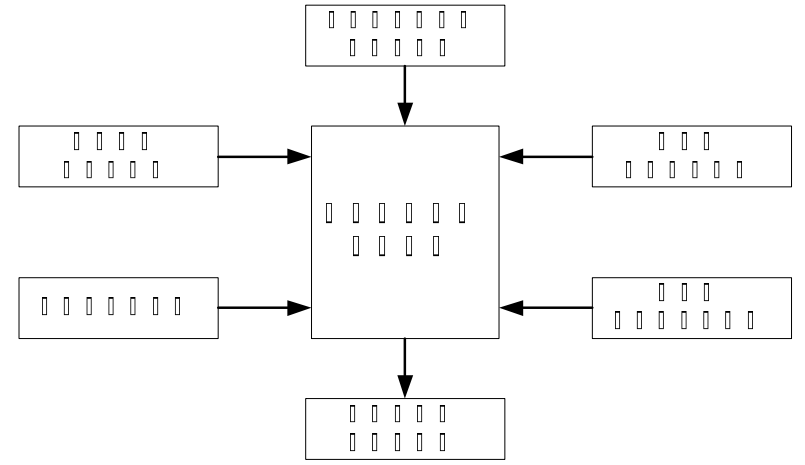

( b ) Time sequence input

Fig.2. the Solar irradiance prediction model

\section{Training network}

RBF neural network training process constantly adjust the process on the network parameters, until the errors between network output and the desired output are sufficiently small, the training process of the network means the end. RBF neural network can be seen as the curve fitting of the multi-dimensional space, for RBF network training can be regarded as to find a best fitting surface 
in a multidimensional space, generally can also understand for using the multidimensional curve to interpolate test data. The three parameters of RBF network need to be solved, the center of the RBF network, the width of the RBF network, and the weight of the RBF network [8].

In this paper, we use GP-RBF algorithm to complete the training of RBF network, shown in Fig. 3, GP-RBF algorithm is a kind of online supervised learning algorithm with structure and parameters of RBF neural network. The structure and parameters of RBF neural network, by using the concept of neuron sensitivity, combined with the method of hyper sphere clustering, establishing a novel increasing and deleting rules to determine on line [9].

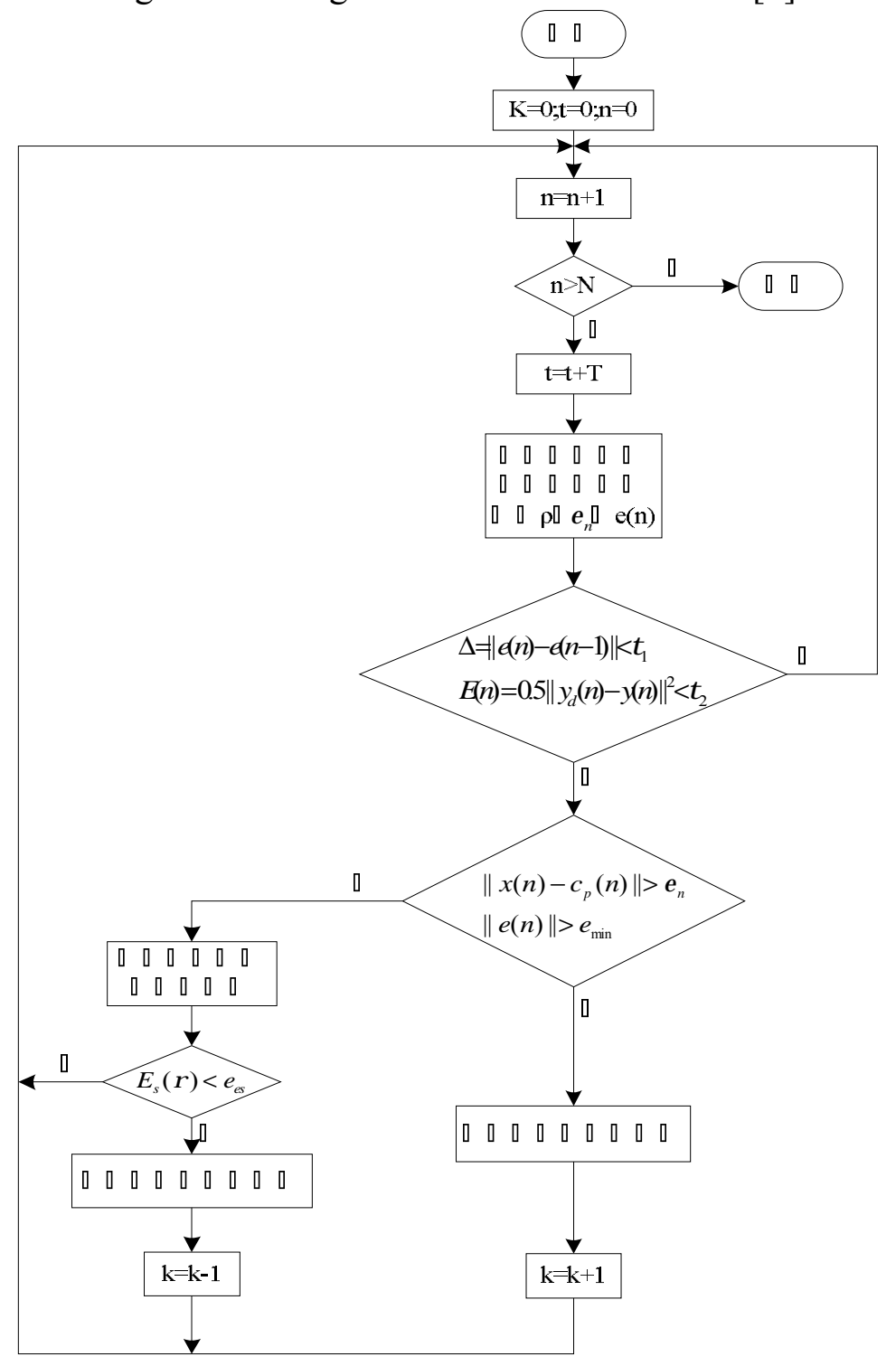

Fig.3. The process of GP-RBF Algorithm

EXIS is extraterrestrial theory irradiance sequence, the UV is ultraviolet sequence, the WT is weather type sequence (sunny 0 , partly cloudy 1 , cloudy 2 , light rain 3 , moderate rain 4 , heavy rain 5 ), ET is the sequence of atmospheric temperature, $\mathrm{Si}$ is solar irradiance sequence, $\mathrm{Si}^{1}$ is the solar irradiance sequence the day before today, $\mathrm{X}$ is the input of the model, $\mathrm{Y}$ is the output of the model. If there is a sample of $\mathrm{M}$ groups, the $X_{M}$ is the input vector group, and $Y_{M}$ is the output vector group. Before the training, $X_{M}$ and $Y_{M}$ should be normalized processing in order to reduce the network error. Network training effects is shown in Fig. 4, implicit layer node number from 0 to 
gradually increasing, when the hidden layer node number is up to 8 , the network target error approaches 0 , which indicates that network output can be a very good function approximation [10].

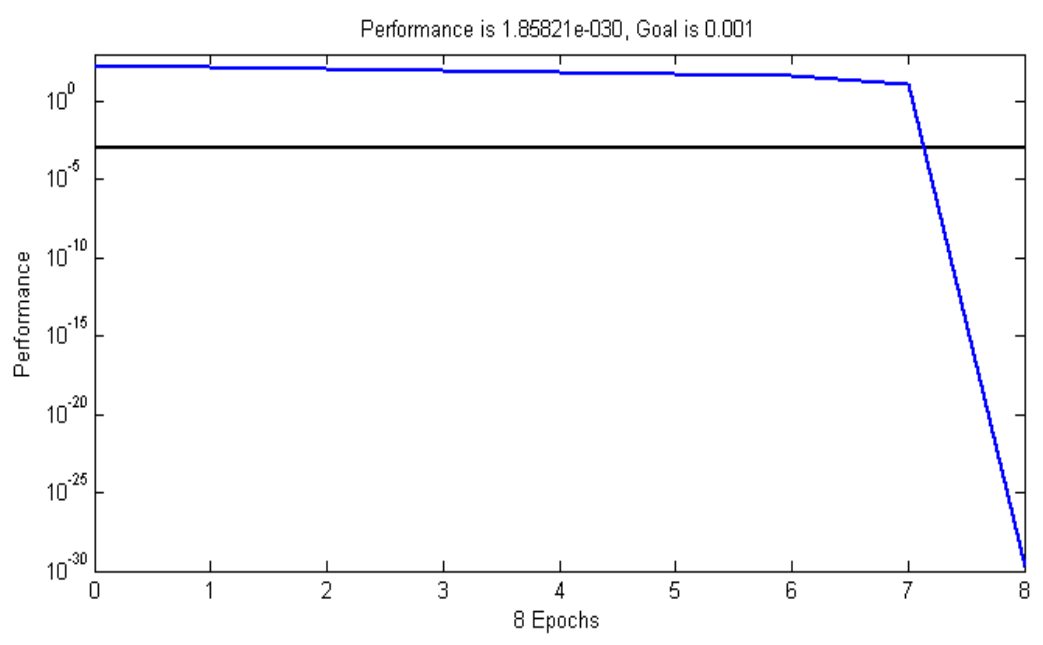

Fig.4. The training effect

\section{Forecast result analysis}

By using hourly input sequence prediction model to predict the solar irradiance, we analyze and predict results through whether hourly weather forecast accuracy. If from 6 a.m. to 7 p.m., there are at least more than 11 period weather forecast, we define weather forecast accurately, if only $8 \sim 10$ period of time, weather forecasts is defined more accurately, only $5 \sim 7$ period of time, weather forecasts is defined less accurately, 4 or less than 4 period of time weather forecasts is defined not accurately.

(1) The weather forecast is accurate

2014.8.5 take the solar irradiance as an example, in the forecast day solar irradiance curve and the real solar irradiance curve are shown in Fig. 5 (a), MAPE $=5.6 \%$.

(2) The weather forecast is more accurate

2014.7.31 take the solar irradiance as an example, in the forecast day solar irradiance curve and the real solar irradiance curve are shown in Fig. 5 (b), MAPE $=8.2 \%$.

(3) The weather forecast is less accurate

2014.8.1 take the solar irradiance as an example, in the forecast day solar irradiance curve and the real solar irradiance curve are shown in Fig. 5 (c), MAPE $=13.8 \%$.

(4) The weather forecast is inaccurate

2014.8.3 take the solar irradiance as an example, in the forecast day solar irradiance curve and the real solar irradiance curve are shown in Fig. 5 (d), MAPE $=21.7 \%$.

As shown in Fig. 5 (a), (b), (c), in accurate, more accurate, less accurate weather forecast, the predicted curve matches the real solar irradiance curve. In individual sessions of the weather forecast which is not accurate, predicted values deviate little. As shown in Fig. 5 (d), in inaccurate weather forecast, the prediction curve and the real solar irradiance curve deviation is not much. That theory of extraterrestrial irradiation value plays a good role in limiting. Using combination of the UV index and weather types parameters can better reflect the real solar irradiance. 


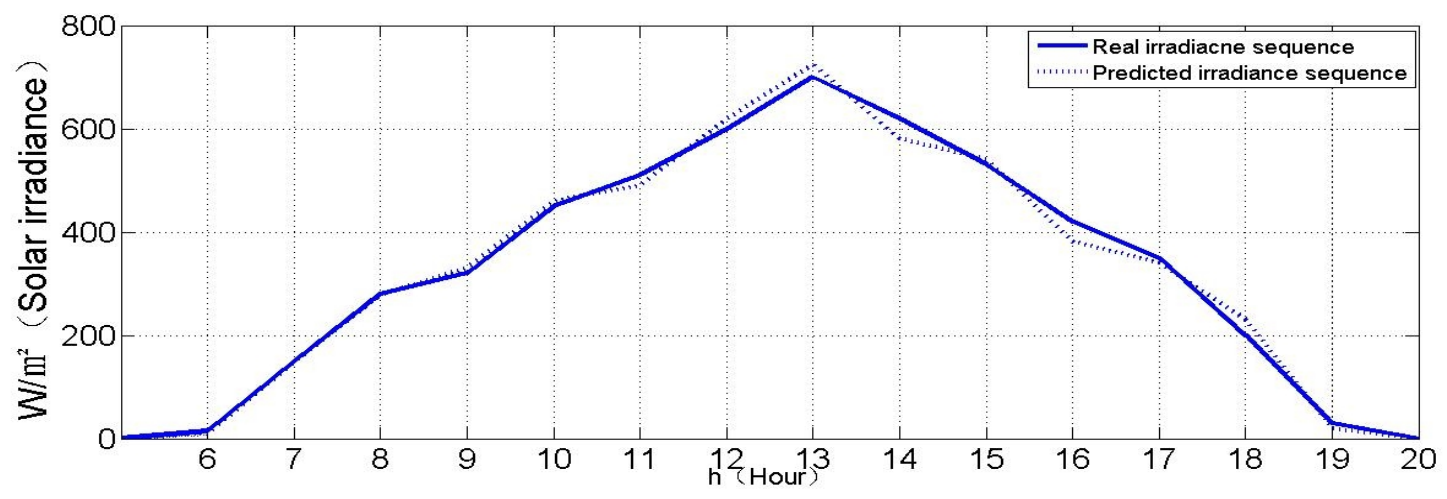

(a)Accurate weather forecast

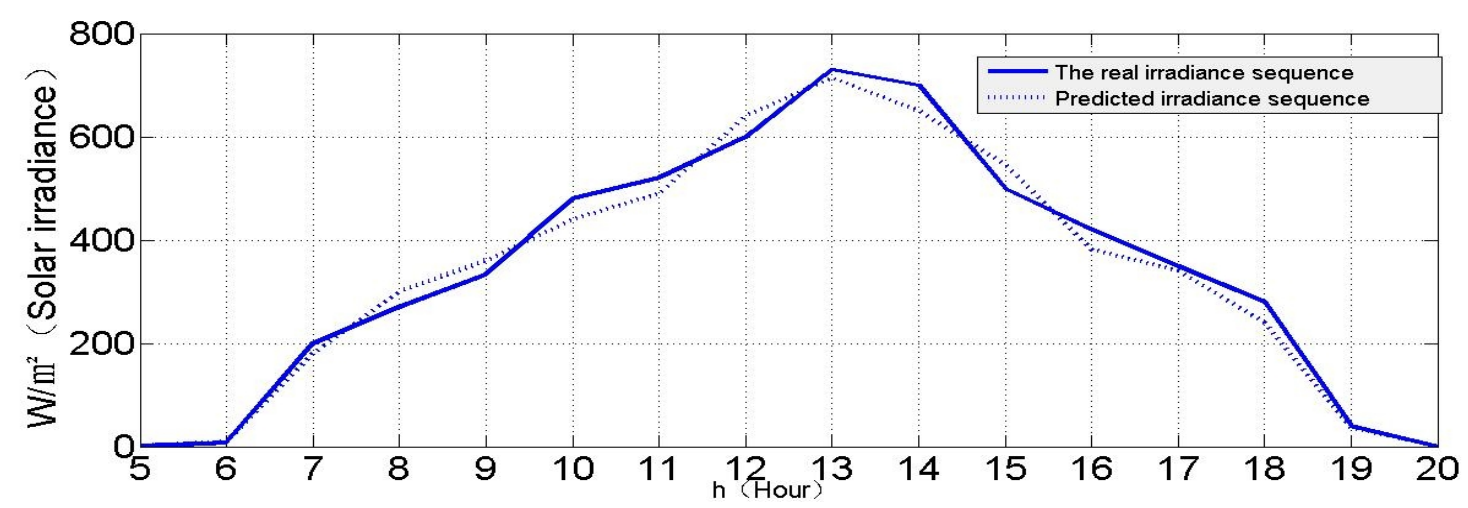

(b)More accurate weather forecast

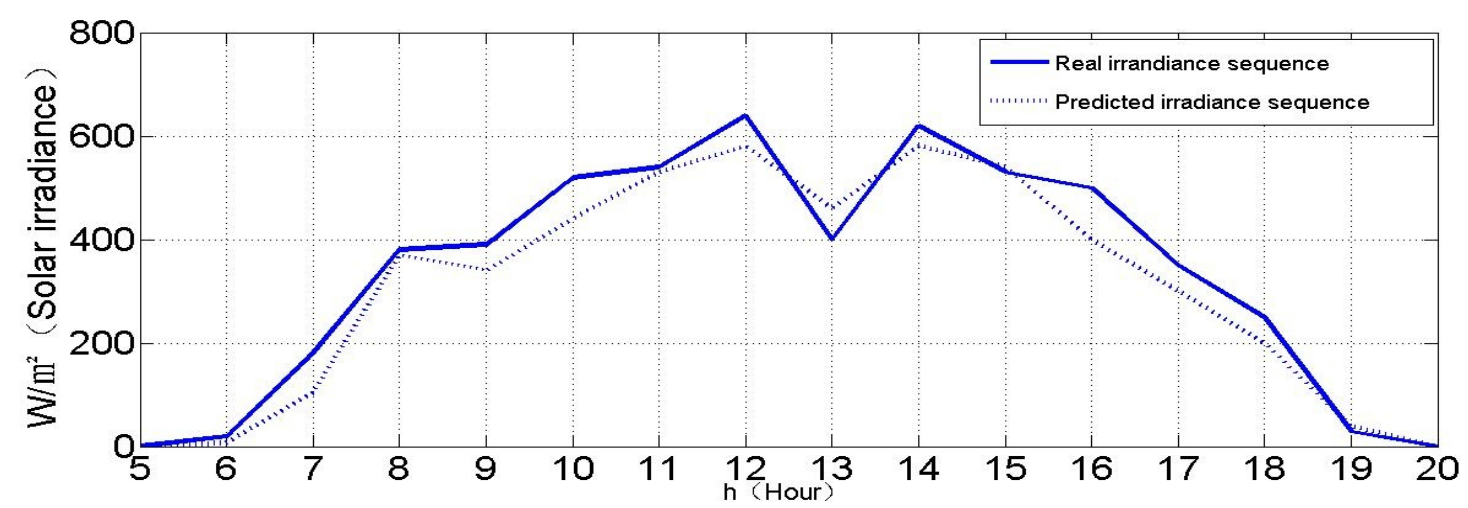

(c)Less accurate weather forecast

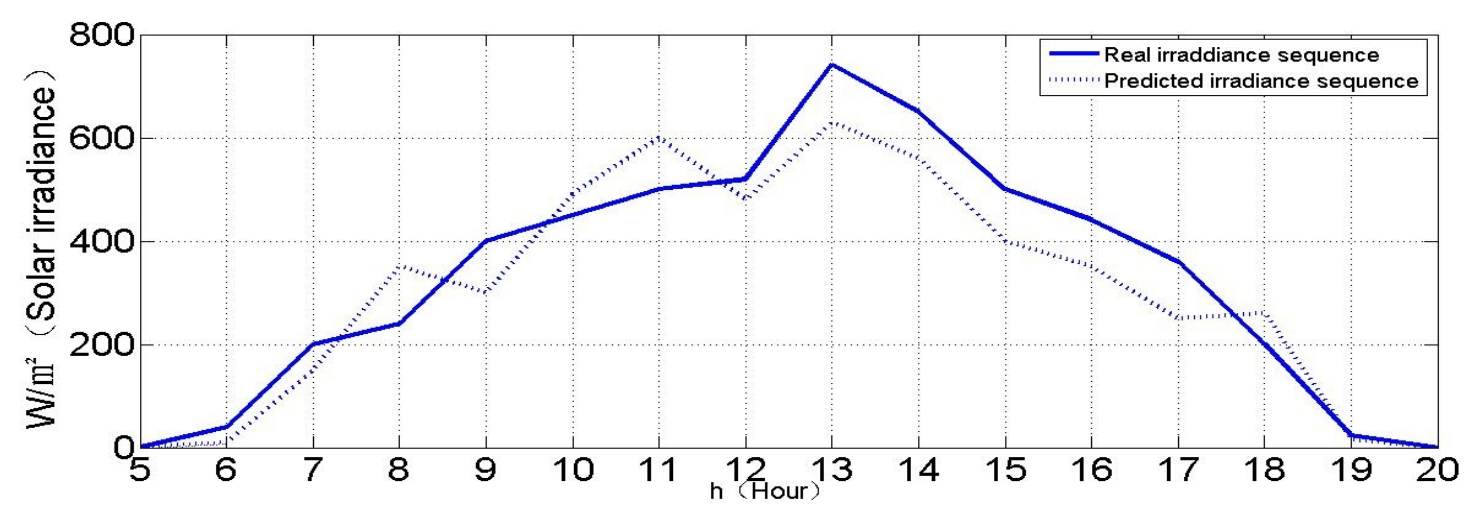

(d)Inaccurate weather forecast

Fig.5. The different situations of solar irradiance curve 


\section{Conclusion}

In this paper, by analyzing the factors affecting the solar irradiance, a solar irradiance forecasting model based on the RBF neural network and input by several of hourly sequences is proposed, five kinds of sequence to establish RBF for training are selected. According to the degree of weather forecast accuracy, the model is established to predict the future solar irradiance in four different conditions for a period of time. The results show that the solar irradiance forecast curve fits the real curve in basic, and has high reliability. Especially in the case of objective forecast is not accurate, forecasting curve deviation is small. As a result, the prediction method is simple, practical, reliable, highly prediction accurate, and has new significance to the forecast of solar irradiance especially the forecasting accuracy of photovoltaic power generation, which is suitable for promotion.

\section{Acknowledgements}

The work in this paper was supported by the National Natural Science Foundation of China (Grant No. 51267001).

\section{Reference}

[1] Yang Si, Haifeng Zhang. Prediction based on neural network of solar irradiance [J]. Qinghai University Journal (Natural science edition), 2013,31(1):15-16.

[2] Zhe Wang. Prediction research of photovoltaic power plant irradiance based on the ANN and periodic time [D]. Beijing: North China Electric Power University, 2012

[3] Shuangxia Chu, Linhua Liu. Analysis of the spectrum of direct solar radiation[J]. Chinese Journal of electrical engineering, 2009,29 (14): 99-103.

[4] Zengqiang Mi, Fei Wang, Guang Yang. ANN prediction in Photovoltaic power plant irradiance and its two dimensional variable metric correction method [J]. Journal of solar energy, 2013,34 (2): 251-254.

[5] Dongdong Lu ,Puyan Zheng, Yanzhou Yuan. Research on the prediction of based on weather forecast for [J]., 2014,15 (2): 43-45.

[6] Dong Qiu, Wenjuan Dai. Prediction model research based on RBF neural network of AOD furnace smelting medium carbon and low carbon ferrochrome of end phosphorus content [J]. Computer measurement and control, 2014,22 (9) 3020-3023.

[7] Changsong Chen. Research on power generation forecasting and energy management of photovoltaic monitoring system [D]. Wuhan: Huazhong University of Science and Technology, 2007

[8] Hui $\mathrm{Hu}$,Guorong Liu. Online robust adaptive neural network tracking control [J]. control theory and applications, 2009,26 (3): 337-338.

[9] Maimouna Diagne Mathieu, David John, Boland. Post-processing of solar irradiance forecasts from WRF modelat Reunion Island [J]. Solar Energy, 2014 (105): 100-107.

[10] Richard Perez, Elke Lorenz, Sophie Pelland. comparison of numerical weather prediction solar irradiance forecasts in the US, Canada and Europ [J]. Solar energy, 2013 (94):306-316. 